\title{
Editor Notes: Neurogenesis and Neurodegeneration: Basic Research and Clinical applications
}

\author{
Henning Ulrich ${ }^{1}$ \\ Published online: 11 January 2022 \\ (c) The Author(s), under exclusive licence to Springer Science+Business Media, LLC, part of Springer Nature 2022
}

The induction of endogenous neurogenesis and engraftment of stem cells for neural repair are demanding tasks in view of the increasing incidence of neurodegenerative diseases. Modern medicine continuously increases life expectancy and subsequently the frequency of neurological diseases, occurring in the ageing population. Efforts have been made to understand molecular mechanisms of neurodegeneration and neuroregeneration. In this regard, embryonic and induced pluripotent fetal and adult stem cells as well as fetal and adult tissue-specific stem cells are employed in in vitro assays to study pathways of neurogenesis as well as for the development of protocols for differentiation into defined neural phenotypes and optimization of the yields of neural differentiated cells. Besides obtaining predifferentiated or fully differentiated cells for transplantation purposes, such stem cell differentiation models can be used to elucidate molecular mechanisms of neurodegenerative disorders and their developmental origin as well as to discover targets for therapy and to probe therapeutic strategies. Furthermore, stem cells are used for engraftment in conditions of brain damage, such as caused by traumatic events and stroke, and as consequences of neurological diseases. Neural stem cells (NSC) have been used for replacement of lost neurons. Functional integration of transplanted cells into neural networks in vivo has been shown by tracing technologies, using fluorescent probes and viral vectors as tracers, as well as by functional analysis based on electrophysiology and optogenetics [1]. While in vivo animal studies have documented the feasibility of stem cell engraftment as possible treatment, functional engraftment of induced pluripotent stem cells (iPSC) into the putamen of a Parkinson's disease patients was demonstrated following 18-24 months of transplantation [2]. Likewise,

\footnotetext{
Henning Ulrich

henning@iq.usp.br

1 Department of Biochemistry, Institute of Chemistry, University of São Paulo, São Paulo, Brazil
}

the treatment of neuronal loss following stroke has also been shown to be promising for cell replacement therapy. Neurons differentiated from human iPSC integrated into neural networks of organotypic neurons of adult human cortex [3]. These data are in line with animal studies, in which human pluripotent stem cells were employed for the treatment of stroke in animal models, such as by transplantation of human embryonic stem cells or IPSC into the sensorimotor cortex, lateral ventricle, hippocampus or striatum [4]. The endogenous environment is determining for neuroregeneration fate and regeneration processes. As an alternative for inserting stem cells for neural network repair, chemotrophic and neurotrophic factors may guide endogenous stem cells to the local of injury for the replacement of lost neurons [5]. Preclinical and clinical studies are underway for the use of stem cells of various origins for the replacement therapy and stimulation of endogenous neurogenesis in ischemic stroke [6,7]. This special issue focuses on the modeling of neurogenesis and neurodegeneration by using iPSC, neurospheres and brain organoids and studying effects of extrinsic and intrinsic epigenetic factors as well as exploiting mechanisms of microglial activation. Topics include the characterization of neural crest cells and iPSCderived neurons as well as neural stemness as the basis of differentiation and its tumorgenic potential. Further optimization of in vitro neural differentiation protocols and the effects of external magnetic fields as determents of differentiation fate are discussed. Mesenchymal stem cell (MSC) activated through electropuncture or stem cell neurogenesis regulation by micro and other non-coding RNAs are discussed for their potential in neuroregeneration. Genetical engineering of MSC as well as inducing them to transdifferentiate into neural stem cells are attractive for the development of therapies to combat neurodegeneration. Oxidative stress reduction following ischemic stroke is an important effect exerted by MSC. Overall, this Special Issue combines in vitro differentiation studies with results obtained in animal models of neurodegeneration. 


\section{References}

1. Palma-Tortosa, S., Coll-San Martin, B., Kokaia, Z., \& Tornero, D. (2020). Neuronal Replacement in Stem Cell Therapy for Stroke: Filling the Gap. Frontiers in Cell and Development Biology, 9, 662636. https://doi.org/10.3389/fcell.2021.662636

2. Schweitzer, J. S., Song, B., Herrington, T. M., et al. (2020). Personalized iPSC-Derived Dopamine Progenitor Cells for Parkinson's Disease. The New England Journal of Medicine, 382(20), 1926-1932. https://doi.org/10.1056/NEJMoa1915872

3. Grønning Hansen, M., Laterza, C., Palma-Tortosa, S., et al. (2020). Grafted human pluripotent stem cell-derived cortical neurons integrate into adult human cortical neural circuitry. Stem Cells Translational Medicine, 9(11), 1365-1377. https://doi.org/ 10.1002/sctm.20-0134

4. Palma-Tortosa, S., Coll-San Martin, B., Kokaia, Z., \& Tornero, D. (2021). Neuronal Replacement in Stem Cell Therapy for Stroke: Filling the Gap. Frontiers in Cell and Development Biology, 9, 662636. https://doi.org/10.3389/fcell.2021.662636
5. Matsubara, S., Matsuda, T., \& Nakashima, K. (2021). Regulation of Adult Mammalian Neural Stem Cells and Neurogenesis by Cell Extrinsic and Intrinsic Factors. Cells, 10(5), 1145. https://doi.org/ 10.3390/cells10051145

6. Zhang, S., Lachance, B. B., Moiz, B., \& Jia, X. (2020). Optimizing Stem Cell Therapy after Ischemic Brain Injury. Journal of Stroke, 22(3), 286-305. https://doi.org/10.5853/jos.2019.03048

7. Ceanga, M., Dahab, M., Witte, O. W., \& Keiner, S. (2021). Adult Neurogenesis and Stroke: A Tale of Two Neurogenic Niches. Frontiers in Neuroscience, 15, 700297. https://doi.org/10.3389/ fnins.2021.700297

Publisher's Note Springer Nature remains neutral with regard to jurisdictional claims in published maps and institutional affiliations. 